\title{
Correction to: Reducing macrophage numbers alleviates temporomandibular joint ankylosis
}

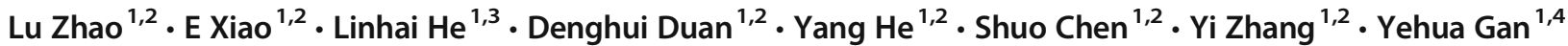

Published online: 18 November 2019

(C) Springer-Verlag GmbH Germany, part of Springer Nature 2019

\section{Correction to: Cell and Tissue Research https://doi.org/10.1007/s00441-019-03087-7}

The article "Reducing macrophage numbers alleviates temporomandibular joint ankylosis", written by Lu Zhao, E Xiao, Linhai He, Denghui Duan, Yang He, Shuo Chen, Yi Zhang and Yehua Gan, was originally published electronically on the publisher's internet portal (currently SpringerLink) on September 2019 with open access.

With the author(s)' decision to step back from Open Choice, the copyright of the article changed on November 2019 to $($ ) Springer-Verlag GmbH Germany, part of Springer Nature 2019 and the article is forthwith distributed under the terms of copyright.

The original article has been corrected.

The online version of the original article can be found at https://doi.org/ 10.1007/s00441-019-03087-7

Yi Zhang

zhangyi2000@263.net

Yehua Gan

kqyehuagan@bjmu.edu.cn

Lu Zhao

lumia_zhao@163.com

E Xiao

xiaoe@bjmu.edu.cn

Linhai $\mathrm{He}$

helinhai07@126.com

Denghui Duan

18910188808@163.com

Yang $\mathrm{He}$

fridaydust1983@163.com
Shuo Chen

chenshuo-88@163.com

Department of Oral and Maxillofacial Surgery, Peking University School and Hospital of Stomatology, \#22 Zhongguancun South Avenue, Haidian District, Beijing 100081, China

2 Laboratory of Oral and Maxillofacial Surgery, Peking University School and Hospital of Stomatology, \#22 Zhongguancun South Avenue, Haidian District, Beijing 100081, China

3 Peking University Hospital of Stomatology First Clinical Division, 37A Xishiku Street, Xicheng District, Beijing 100034, China

4 Central Laboratory, Peking University School and Hospital of Stomatology, \#22 Zhongguancun South Avenue, Haidian District, Beijing 100081, China 